Miša Glišič

Študentka Oddelka za germanistiko

Filozofska fakulteta, Univerza v Mariboru

glisic.misa@gmail.com
UDK 821.163.6(436.5).09-31Haderlap M.

DOI: $10.4312 /$ vestnik.6.107-122

\title{
DIE AUTHENTIZITÄT IN MAJA HADERLAPS ROMAN ENGEL DES VERGESSENS
}

\section{EINLEITUNG}

Maja Haderlap schafft in ihrem Romandebüt Engel des Vergessens eine soziohistorische Distanz, die durch zwei Kulturen geprägt ist. Dabei darf man nicht vergessen, dass die Sprache ein wichtiger Teil der Kultur ist. Der Roman wurde auf Deutsch geschrieben, obwohl die Muttersprache der Autorin Slowenisch ist. Darin werden nicht nur die Unterschiede des Zusammenlebens von verschiedenen Kulturen, sondern auch kulturelle und historische Konflikte deutlich gemacht. Im Zentrum des Geschehens steht der interkulturelle Konflikt zwischen Slowenen und Österreichern/Deutschen zur Zeit des Zweiten Weltkriegs in Kärnten: Die Hauptfigur ist ein Mädchen, das im Laufe des Romans zu einer jungen Frau heranwächst, die sich mit zwei Ländern und Kulturen verbunden fühlt und doch nirgends wirklich heimisch ist. Die Erinnerungen, Gedanken und Gefühle des Kindes sind noch immer lebendig. Die Schriftstellerin muss selbst als Tochter am eigenen Leib erfahren, dass die tiefen Spuren des Zweiten Weltkriegs in der Bevölkerung geblieben sind. Die Ich-Erzählerin erfährt Wärme und Geborgenheit vor allem durch die Liebe der Großmutter. ${ }^{1}$ In den Vordergrund tritt auch die Emanzipation des Denkens, wodurch die Befreiung aus gesellschaftlichen Abhängigkeiten und der Gewinn von Autonomie deutlich werden. Der Text kann auch als ein kollektives Bewusstsein der Gesellschaft bzw. der Kärntner Slowenen betrachtet werden: die diachrone Ebene konzentriert sich auf die historischen Ereignisse. Eine wichtige Rolle spielt dabei auch das Unterbewusstsein. Die Bedeutung der Charakterisierung der Figuren kann durch psychoanalytische Deutung entschlüsselt werden. Die ,Stickigkeit“ des Lepena-Grabens in der Nähe von Eisenkappel, wo der Roman hauptsächlich spielt, hat einen großen Einfluss auf die Umgebung und die Bewohner. Das literarische Werk von Maja Haderlap drückt die ganze Dualität der schriftstellerischen und menschlichen Identität, zugleich aber auch die

$1 \quad$ Nach der Philosophie von Immanuel Kant wird der Mensch erst durch Liebe, Empathie und moralische Sensibilität kultiviert und erst dadurch auch zur Bildung fähig (Winkel, Reiner, 2005: Am Anfang war die Hure. Baltmannsweiler). 
Besonderheiten des interkulturellen Dialogs in dem Gebiet des österreichischen Kärntens aus. Dadurch entsteht im Werk eine ausdrückliche Authentizität, mit der wir uns im Weiteren befassen werden.

\section{DAS FREMDE}

Der Roman Engel des Vergessens spricht über die Familiengeschichte und das Leben der Kärntner Slowenen, die als Figuren im Roman komplex erscheinen. Engel des Vergessens ist die Erinnerung an die Grausamkeiten des Nationalsozialismus in Österreich. Der Roman bietet einen tiefen Einblick in das Leben der Kärntner Slowenen, die im Zweiten Weltkrieg Opfer des Totalitarismus waren. ${ }^{2}$

Die heutigen Kulturen seien innerlich durch Pluralisierung der Identitäten und äußerlich durch die Überschreitung der eigenen Grenzen gekennzeichnet. Homi K. Bhabha weist auf die Kategorien des Eigenen und Fremden hin (Bhabha, Homi. K., 1994: The Location of Culture. Routledge). Bhabha spricht sich für eine Hybridität aus, in der die Differenz zwar aufrechterhalten bleibt, jedoch ohne eine vorausgesetzte Hierarchie. Auch im Bereich von individueller Mehrsprachigkeit entstehen hybride Sprachformen. In unserem Roman steht die slowenische Sprache im Bezug zu der deutschen Sprache.

Alois Wierlacher stellte fest, dass die Beziehungen zwischen Eigenem und Fremdem zu berücksichtigen sind. Das Fremdverstehen geht immer aus unserer Selbsterkenntnis hervor (Wierlacher, Alois, 1985: Das Fremde und das Eigene. Prolegomena zu einer interkulturellen Germanistik. München). Wenn wir uns selbst nicht verstehen, können wir auch andere nicht verstehen. Wierlacher spricht von Fremdheit als einem Interpretament der Andersheit, die vom Betrachter immer durch den Filter eigenkultureller Vorverständnisse gesehen wird. Die Protagonistin lernt das Eigene besser auf der Folie des Fremden verstehen, das jedoch erst durch die Begegnung sichtbar wird. Selbst die individuelle, scheinbar unmittelbare Wahrnehmung des Anderen als Fremden ist soziokulturell geprägt.

Das Eigene bildet in dem Roman die Zugehörigkeit der Erzählerin zu den Kärntner Slowenen und das Fremde die deutsche Sprache und das, was mit dieser Sprache hervorgebracht wird. Die Ich-Erzählerin kommt automatisch durch den Besuch des Gymnasiums in Klagenfurt mit der deutschen Sprache in Berührung. Dadurch wird der Zwiespalt der Protagonistin deutlicher. Es kommt zur Kulturverflechtung. Bei der Auswahl der deutschen Sprache wird das Fremde in seiner trennenden und vereinigten Andersheit erkennbar gemacht. Das Fremdverstehen führt letztendlich zur Introspektion

2 Autobiografische Züge sind das bedeutsamste Merkmal des Romans. Die Autorin wollte keine Fiktion schreiben, sondern durch ihre eigene Geschichte zur größeren Authentizität des literarischen Werks beitragen, was ihr sehr gut gelungen ist. 
der Protagonistin. Die Schulbibliothek erhält über die Bereitstellung von Büchern und damit die Vermittlung von Historie und Kultur auch eine weitere Bedeutung für die IchErzählerin. Durch den Kontakt mit der deutschen Sprache, Literatur und Kultur wird die Bildung des Individuums ermöglicht:

Ich gehe durch die Schule der Minderheitenfeststellung in Kärnten und begreife die Aussage der Parole, die auf den Plakaten prangt: Wähle Deutsch, wenn du kein Slowene sein willst! Das Slowenische ist also etwas Unerwünschtes im Land, denke ich und entscheide mich für das öffentlich Geringgeschätzte, weil es in meinen Augen und in den Augen der Menschen, mit denen ich lebe, eine Bedeutung hat und weil ich das erste Mal begreife, was mit dem Wort Zugehörigkeit gemeint sein könnte. $(\mathrm{EdV}, 143)^{3}$

Dieses Zitat belegt, dass die Sprache ein Ausweis der Identität eines jeden Menschen ist. Sie lässt den Menschen sich selbst und die anderen finden. Die Grenzen zwischen objektiver und fiktionaler Realität verschwinden und die literarischen Darstellungen im Werk, dazu gehört auch die Repräsentation von Fremdheit, werden vom Leser als wirklich existent angesehen.

\section{DIE FRAGE DER IDENTITÄT UND DER MULTIKULTURALITÄT}

Die multikulturelle Kommunikation findet oft auf der interlingualen Ebene statt und ist Folge der ständigen Präsenz von mehreren Kulturen im Leben des Menschen. Das Vorhandensein von mehreren Kulturen kann jedoch nicht isoliert betrachtet werden; zwischen den Kulturen entstehen verschiedene Kontakte, wie auch Konflikte. Diese äußern sich im Gefühl der Entfremdung als Ergebnis des entfremden Verhältnisses einer Kultur im Bezug auf die andere, in der unerwünschten Verflechtung der Elemente von verschiedenen Kulturen, in der zunehmenden Hybridität und in dem Eindringen von unerwünschtem Fremden in die eigene Kultur (Grosman 2004: 17 ff.). Besonderes Interesse erwecken die interlingualen Kontakte, weil der Gebrauch der Sprache die Kultur und ihre Erhaltung verkörpert. In Bezug auf die Realität können wird das am folgenden Beispiel aus dem Roman sehen:

In der Vergangenheit ist meine Familie zu einer national handelnden Familie geworden, als sie sich gezwungen sah zu reagieren, weil sie durch ihre Zugehörigkeit gefährdet war, weil es um ihr physisches Überleben ging. Entweder

3 Maja Haderlap: Engel des Vergessens. Roman. Göttingen: Wallstein 2011. Im Folgenden EdV und Seitenangabe. 
die Sprache und die Kultur vergessen und im Deutschen aufgehen, oder sich wehren und die verheerenden Folgen ertragen. (EdV, 221)

Moderne Literaturtheorie erforscht die Verhaltensweise der Macht und verschiedene Rollen der Literatur der neuzeitlichen nationalen Minderheiten, die ihre Lebensweise geändert haben und im „Fremden“ leben. Es geht um die Erforschung von „hybriden Identitäten“, einer Art von „Patchwork“ (Hoffmann 2006: 13).

Mehrsprachigkeit hat einen großen Einfluss auf die hybride Identitätsbildung. Sie sorgt für die Entstehung sogenannter kodierter, komplexer Identitäten, die die Literatur thematisiert und die in Form einer symbolischen Sprache im Kanon zu finden sind. Maja Haderlap erzählt kühl analytisch und doch achtsam von einer Gemeinschaft, die stolz ist auf ihre Eigenart und auf die Tragödien der Vergangenheit - und zugleich verbittert über ihr Los, ausgeschlossen zu sein.

Im Roman Engel des Vergessens können wir besondere Formen der schriftstellerischen Auseinandersetzung mit der eigenen doppelten persönlichen Identität betrachten. Maja Haderlap verweist auf die Multikulturalität, die sich in der traumatischen Kriegsgeschichte der Kärntner Slowenen zeigt. Das Phänomen der Multikulturalität in der Literatur haben schon Strutz und Zima beschrieben. Sie sind zur Erkenntnis gekommen, dass individuelle Autoren mit dem Gebrauch von Sprache, die nicht ihre Muttersprache ist, oft versuchen, sich von einer Art von Besessenheit mit der Muttersprache zu befreien (Strutz, Zima 1996). Wie Maja Haderlap sagt, hält sie die deutsche Sprache auf Distanz zum Schmerz (Mayer, Norbert, 2012: Haderlap: „Deutsch hält mich auf Distanz zum Schmerz“. In: Die Presse.com). Sie hat ihren Roman auf Deutsch verfasst, weil sie wollte, dass sich die Mehrheit der Bevölkerung mit dem Spiegelbild der historischen Ereignisse von zwei Kulturen und Sprachen in einem bestimmten geographischen Gebiet auseinandersetzt. Somit erhoffte sie sich eine Reflexion über die geschichtliche Diskriminierung der Kärntner Slowenen. Auch Silvija Borovnik sieht die Wahl der deutschen Sprache als eine Möglichkeit, dass die vernachlässigte Geschichte auch denen zugänglich wird, für die sie bestimmt ist - in der slowenischen Sprache würde sie aber wahrscheinlich vergessen bleiben (Borovnik 2012: 198).

Jedoch baut die Erzählerin ihre Identität zunächst auf der slowenischen Sprache auf. Die Familienmitglieder sind religiös, singen katholische Lieder auf Slowenisch und kommunizieren miteinander in slowenischer Sprache; die intimsten Beziehungen werden durch die slowenische Sprache geprägt. Davon zeugen auch Zitate in der slowenischen Sprache, die einen besonderen Stellenwert im Vergleich zum deutschen Text haben:

Mutter betet mit mir sveti angel varuh moj, bodi vedno ti z menoj, stoj mi dan in noč ob strani, vsega hudega me brani, amen und sagt, dass Engel in die Seele eines Menschen blicken und ihre geheimsten Gedanken lesen können. $(\mathrm{EdV}, 13)$ 
Die Sprache ihrer Bildung war Deutsch, die Sprache ihrer Kindheit ist aber Slowenisch geblieben. Dieser Roman ermöglichte ihr nicht nur eine andere persönliche, sondern auch eine andere sprachliche Identität. Verschiedene Forscher, die sich mit dem Phänomen der literarischen Polyphonie beschäftigen, behaupten, dass der Gebrach mehrerer Sprachen und der Ausdruck mehrerer Kulturen im literarischen Werk nicht nur die Koexistenz der Vielfalt, sondern auch die historischen und multikulturellen Konflikte zum Ausdruck bringt.

\section{DIE ROLLE DER FRAU UND DIE SUCHE NACH SICH SELBST}

Im Roman tritt auch die Rolle der Frau in den Vordergrund, vor allem in der Figur der Großmutter. Ihre Geschichte hat eine besondere und entscheidende Bedeutung im Verlauf des Romans und auch ihre assoziative Erzählweise hat einen großen Einfluss auf das Enkelkind:

Großmutters Schlafzimmer ist ein Gedächtnisort, eine Königszelle, in der alles in eine milchige Flüssigkeit eingetaucht scheint, eine Brutzelle, in der ich mit Großmutters Nährflüssigkeit gefüttert werde. In dieser Keimzelle werde ich, wie ich erst Jahre später begreifen werde, geformt. (EdV, 117)

Die Kriegsgeschichte wird aus der Perspektive der Frau erzählt und veranschaulicht. Die Großmutter hat die Rolle einer einfachen Frau auf dem Lande, der Mutter und ist zugleich Zeugin der diachronen Geschehnisse. Durch ihr Erzählen können wir eine Empathie für die Frauen, die in das Konzentrationslager in Ravensbrück deportiert wurden, entwickeln. Die Frauen wurden misshandelt, beleidigt, gequält, ausgehungert, ermordet und von ihren Kindern getrennt:

So wenig Brot gab es zu essen im Lager, so wenig, deutet sie mit dem Daumen und dem Zeigefinger die Größe der Brotstücke an, die den Häftlingen zugeteilt wurden. Es musste reichen für einen Tag, manchmal für zwei. Später bekamen wir nicht einmal das, sagt sie, und haben das Brot fantasiert. Ich blicke sie an. Sie sagt, wie sie immer sagen wird, je bilo čudno, es war befremdend, sagt sie und meint, es war schrecklich, aber grozno fällt ihr nicht ein. $(\mathrm{EdV}, 10)$

Das Bild der Großmutter wird durch ihre innere Stärke und ihren Stolz geprägt. Das Durchhaltevermögen lernt das Kind vor allem in der Beziehung zur Großmutter; die Ich-Erzählerin ist eine selbstständige, vollkommen emanzipierte Frau, die ihren eigenen Weg geht und ihre Ziele verfolgt. Die Mutter des Kindes dagegen verfolgt die sogenannte 
„3K-Regel“ - die Frau wird durch kochen, Kinder und die Kirche definiert. Die Geschichte im Roman ist dadurch nicht mehr nur die Geschichte der Männer und des Krieges, sondern zugleich die Geschichte der Emanzipation der Frau und des alltäglichen Lebens. Die Großmutter ist eine psychisch außerordentlich starke Frau. Sie erzählt realistisch über die Vergangenheit, die in Verbindung zu ihrer detaillierten Geschichte mit vielen von ihren Erlebnissen steht:

Sie sagt, sie habe auch Essen gestohlen für sich und die anderen, sie habe nach jeder Kartoffelschale gesucht, nach allem, was essbar schien, damals, als sie die Kessel gewaschen hat, das war noch ein Glück, sagt sie, dass sie dahin gekommen sei, in die Küche, im Lager, ich weiß. (EdV, 6)

Sie spricht über die Gewalt des Faschismus, dessen Opfer die slowenischen Familien in der Nähe von Eisenkappel waren. Sie erinnert sich an die Internation der Nachbarn, die Brandstiftungen der Häuser und die Ermordung vieler Familien. Sie sorgt für die Menschen und nimmt sie in Schutz. Die Großmutter zählt die Geschichten von Frauen, die nicht nach Hause zurückkehren konnten, auf (Haderlap, Maja, 2012: Engel des Vergessens. Roman. Göttingen. S. 120 - 130). Die Frauen wurden gezwungen, die Männerarbeit zu übernehmen und ihre Männer und Söhne zu ersetzen. Die Frauen waren einer kontinuierlichen Gefahr ausgesetzt, weil die Nationalsozialisten ihre Machtlosigkeit und ihre fragile Natur ausgenutzt haben. Die Frauen wurden von Tag zu Tag stärker, entschlossener und mutiger. Viele Frauen von der Umgebung halfen und boten den Partisanen Hilfe an.

Die Großmutter verbringt viel Zeit mit ihrer Enkelin und ist zugleich ihre Autorität. Die Mutter dagegen wird als eine unglückliche und verzweifelte Frau beschrieben. Auch die Religion der Großmutter wird als Gegensatz zur Religion der Mutter dargestellt:

Den Glauben an Gott müsse man im Herzen tragen, sagt Großmutter, es genüge nicht, ihn in der Kirche zur Schau zu stellen. Auf die Kirche sei kein Verlass, findet sie, man könne ihr nicht trauen. (EdV, 27)

\section{DIE EMOTIONALITÄT}

Der Roman ist komplex, viele Phänomene werden illustriert und unter die allgemeinsprachlichen Begriffe der Gefühle und Emotionen subsumiert. Die Protagonistin ist ein Instinkt-Wesen mit Empfindungen, Leidenschaften, Phantasmen und Affekten. Die Liebe der Großmutter zur Erzählerin, das Interesse bzw. die Zuneigung zur Landschaft, die Freundschaft und die Selbsterkenntnis geben dieser Literatur einen besonderen Wert. Auch die Beschreibungselemente, die Dejan Kos in seiner Theoretischen 
Grundlage der empirischen Literaturwissenschaft einführt, nämlich die Komponenten, bzw. Ordnungsfaktoren, stabile Interaktionsmuster zwischen den Systemkomponenten sowie die Funktionen (vgl. Kos 2003, 46 - 57.) lassen sich in verschiedenen Forschungsfeldern, auch dem der Emotionen, konkretisieren. Diese Komponenten werden sowohl synchron wie auch diachron kulturell kodiert. Diese Theorie bestätigt sich auch in dem hier behandelten Roman.

Die Protagonistin erkennt schon als Kind sofort, wenn es der Großmutter schlecht geht und sie zu emotional wird. Mit der Beobachtung ihrer Körpersprache dekodiert das Kind „die Geheimnisse der Sprachzeichen“. Die Vergangenheit hat einen Einfluss auf das Kind und auf die gegenwärtige Situation, was das folgende Beispiel bestätigt:

Meine Sinne werden Großmutters Vibrationen auf die Welt übertragen und die Möglichkeit der Zerstörung in allem sehen. (EdV, 27)

Die Authentizität des alltäglichen Lebens wird durch verschiedene Funktionen sichtbar. Im Roman können didaktische und hedonistisch-emotionale Funktionen der Literatur erforscht werden. Ein Beispiel für die didaktische Funktion wäre die religiöse Erziehung der Mutter. Die Kinder mussten regelmäßig die Kirche besuchen und einen sieben Kilometer langen Weg zu Fuß gehen. Die hedonistische Funktion ist bei dem Kind, das von Süßigkeiten schwärmt, zu sehen. Das Essen von Süßigkeiten verbindet die Erzählerin mit Freude und Glück: Wie ein klebriger Lichtbrei strömt der Honig über meinen Gaumen und erfüllt mich mit Entzücken. (EdV, 23)

Die emotionale Funktion wird im Verhältnis Kind - Großmutter sichtbar. Das Kind wohnt bei der Großmutter und schläft bei ihr im Bett. Das Kind verfolgt sie auf Schritt und Tritt. Die Antworten auf seine vielen Fragen bekommt das Kind immer von der Großmutter. Ihre Beziehung wird am besten mit dem folgenden Zitat beschrieben:

Kaum setzt sie sich in Bewegung, folge ich ihr. Sie ist meine Bienenkönigin und ich bin ihre Drohne. Ich habe den Duft ihrer Kleidung in der Nase, den Geruch nach Milch und Rauch, einen Hauch von bitteren Kräutern, der an ihrer Schürze haftet. Sie gibt mir den Rundtanz vor und ich tänzle ihr nach. Ich passe meine kleinen Schritte ihren schleppenden an, ich summe eine zarte Melodie aus Fragen und sie spielt den Bass. (EdV, 7)

Sie kämmen sich gegenseitig die Haare und sind lieb zueinander. Durch die Großmutter lernt die Protagonistin nutzvolle Dinge über das Leben. Man muss immer offen für Gäste sein und immer ein Stück Brot zu Hause haben und das Essen nicht verschwenden. Die Menschen muss man respektieren und ihnen zuhören. Die Großmutter bringt dem Enkelkind bei, dass man an die Sprache glauben müsse und sie respektieren sollte. Die körperliche Liebe ist ein Bestandteil des Verhältnisses. Das 
Kind wird liebevoll behandelt, gestreichelt, umarmt und mit Süßigkeiten verwöhnt. Geheim darf es aus dem Fläschchen trinken, obwohl es schon zu groß ist. Auch das Leben mit den Tieren auf dem Hof hat bestimmte Konnotationen, die eine emotionale Bedeutung haben.

\section{HISTORISCHE FIKTION}

In Roman Engel des Vergessens können Typologie und Poetik des historischen Erzählens nachgewiesen werden. Die dominante Heteroreferentialität bzw. der ausgeprägte Wirklichkeitsbezug wird bei den Briefen, die ein bestimmtes Datum zeigen und die die Großmutter aufbewahrt hat, deutlich:

9. 3. 1946, Liebe Mitzi! Besten Dank für deine lieben Zeilen, freut mich sehr, dass du so gut nach Hause gekommen bist [...] $(\mathrm{EdV}, 130)^{4}$

Bei der Relationierung und Gestaltung der Erzählebenen gibt es einen hohen Grad an Explizität der erzählerischen Vermittlung. Der Text kann als Traum, Märchen, Mythos und Historie angesehen werden. Bei der Einfühlung in die Vergangenheit ist der Text ein Geflecht aus Träumen, Erinnerungen und Gedanken. Die Auswirkung der Erinnerungen ist bei der Großmutter zu sehen, als sie ein nervöser Hunger überkommt, wenn sie über die Vergangenheit im Lager erzählt und die Essensrationen erwähnt.

In Roman gibt es zwei wichtige geografische Gegensätze. Wien als Studienort wird als eine Art von Befreiung aus dem Graben und als das intellektuelle Zentrum für die weitere Ausbildung angesehen und die Umgebung von Eisenkappel wird detailliert als Belastung bzw. als eine Grabenfalle beschrieben:

Die heimatlichen Hügel haben sich in eine Falle verwandelt, die jeden Sommer nach mir greift und zuschnappt. Ich kann meinen Geburtsort immer weniger mit meinem Leben in Verbindung bringen und überlege, mir Notsteige zu bauen, um meine Zuversicht aus dem Graben schmuggeln zu können. Noch versuche ich in der Landschaft Trost zu finden, einen Lebensort in ihr aufzuspüren, der mich nicht bedroht. $(\mathrm{EdV}, 190)^{5}$

4 Dass der Roman auf wirklichen Geschehnissen beruht, bestätigen die Aufzeichnungen der Großmutter, die die Autorin als Basis für das Entstehen des Romans verwendet hat. Mit dem Roman erfasste Maja Haderlap die Vergangenheit ihrer Familie. Sie fühlte sich verantwortlich für das Nacherzählen der Geschichten ihrer Großmutter. 5 Im Zentrum stehen die Familienverhältnisse und das Nacherzählen der Geschichten ihrer Großmutter, die eine große Auswirkung für das weitere Leben hatten. 
Beim Zeitbezug findet man eine dominante Vergangenheitsorientierung und eine linear-chronologische Anordnung des Geschehens. Bei den Vermittlungsformen werden die Figuren als Beobachter historischer Ereignisse betrachtet. Der Vater war als Kind ein Beobachter der historischen Ereignisse, denn er hatte zugesehen, wie seine eigene Mutter ins Konzentrationslager deportiert wurde. Die Partisanen sind im Gedächtnis der Bevölkerung verankert. Maja Haderlap hat der Geschichte der Kärntner Partisanen eine Stimme gegeben, meinte die österreichische Literaturwissenschaftlerin und Jurorin Daniela Strigl, die Haderlap für den Bachmann-Wettbewerb vorgeschlug. Sie meint, dass Maja Haderlap darüber bedächtig, mit großer Genauigkeit und ohne Hass geschrieben hat (ORF Kärnten, Tage der deutschsprachigen Literatur, Ingeborg-Bachmann-Preis 2011). Das bestätigt sich in der Erzählensweise der Autorin, die die Geschichte der Erinnerungen ohne Beschuldigung und Polemik erzählt.

Beim Zeitbezug beobachten wir das Oszillieren zwischen Vergangenheits- und Gegenwartsorientierung. Die Protagonistin begibt sich mehrfach in ihre Kindheit und hört ihre innerlich bekannte Stimme, die sie in die Gegenwart begleitet. Es gibt eine linear-chronologische Anordnung des Geschehens. Bei den Vermittlungsformen werden Figuren als Opfer des historischen Geschehens betrachtet. ${ }^{6}$ Die Folgen des Krieges sind am besten bei dem Vater zu sehen. Der Raum dient als Schauplatz geschichtlicher Ereignisse. Figuren werden auch als historische Akteure angesehen. Die Großmutter und der Vater haben aktiv am Krieg teilgenommen. Der Mensch wird somit von der soziologischen Perspektive in seinem Handeln bestimmt. Die Zeitungen, Reisepässe und Tagebücher sind der Beweis, dass die Geschichtsdarstellung als Medium kultureller Erinnerungen dargestellt wird.

Bei der Pluralität und Relativität der Zeiten geht es um reale und fiktive Zeit. Das sehen wir aus den Träumen. Träume haben meistens eine Fluchtfunktion. Wenn die Protagonistin träumt, geht es um fiktive Ereignisse, und wenn sie aufwacht, kehrt sie wieder in die Realität zurück:

Ich bin zur Gruppe angewachsen und träume einen Traum, in dem ich in der ersten Reihe einer Prozession von Slowenen gehe. Ich kenne die Menschen, aber sie scheinen mich nicht zu sehen, obwohl ich nackt bin. Im Moment, in dem ich meine Nacktheit bemerke, denke ich, es kann mir nichts mehr geschehen, weil ich tot bin, niemand kann mir etwas anhaben, denn ich bin unsichtbar geworden. (EdV, 143)

Auch der Mythos des christlichen Glaubens ist im ganzen Werk präsent. Die Symbolik des Engels ist eine Art Schutz und Verinnerlichung für die Protagonistin. Wir sehen,

$6 \quad$ Authentizität der historischen Ereignisse ist noch heute zu sehen. Die Auswirkungen der Erfahrung von Verfolgung und Internierung während der NS-Zeit prägen bis heute das Leben der Kärntner Slowenen, und sie geben die erlittenen Traumatisierungen bewusst und unbewusst an ihre Kinder und Kindeskinder weiter. 
dass die Literatur ein Ausdruck der menschlichen Psyche ist. Die Ausdrucksformen des Unbewussten sind im literarischen Werk zu erschließen. Literatur ist das Ergebnis von Sublimierung einer Wunscherfüllung. Die Ich-erzählerin kämpfte mit ihrer Kindheit und ein Gefühl der Schuld hat sie immer begleitet, weil sie als Kind den Tod als allgegenwärtig gesehen hatte. Die Erinnerungen stellen gewisse Szenen in den Vordergrund und verblassen andere. Die Menschen werden wegen der ständigen Verdrängung verzweifelt und verschlossen. Das Leben ist für sie nicht mehr lebenswert. In der Umgebung werden sie „krank“. Sie können das Erlebte nicht vergessen und spüren den Schmerz der Opfer des Krieges und der Nachkriegszeit. Sie sind nicht in der Lage sich vom Erlebten zu befreien. Zwischen den entgegengesetzten Zeitaltern, also zwischen der Vergangenheit und der (historischen) Gegenwart besteht ein Zeitkonflikt. Der Wald ist ein Symbol für die grausamen Geschehnisse, die man noch jetzt spüren kann. Die Natur dagegen wird revisionistisch beschrieben:

In den Wald zu gehen hieß eben »nicht nur Bäume zu fällen, zu jagen oder Pilze zu sammeln«. Es hieß, sich zu verstecken, zu flüchten, sich den Partisanen anzuschließen und Widerstand zu leisten. Wem die Flucht nicht gelang, dem drohten Verhaftung, Tod, Konzentrationslager. $(\mathrm{EdV}, 75)^{7}$

Die Geschichte der Kärntner Slowenen wird im Roman auch privatisiert. Die Ich-Erzählerin fühlt sich mit der Geschichte verbunden. Diese hat einen großen Einfluss auf sie und bildet zugleich einen Teil von ihr. ${ }^{8}$ Im literarischen Werk ist auch die Pluralisierung von Geschichte zu sehen. Der Roman bietet ein Geschichtsbild über Kärnten und die KZ-Lager. Auch die Klarheit in der Gliederung ist zu sehen. Im Roman gibt es eine klare Struktur und Absätze sowie einen logischen linearen und chronologischen Ablauf der Ereignisse. Bei der zeitlichen Linearität sind Sprünge bedeutsam. Dazu gehören die Erzählungen der Großmutter über die Vergangenheit. Der Tod der Großmutter wird schon als zukünftiges Geschehen beschrieben.

Die Geschichte wird auch aus der Perspektive der Ernsthaftigkeit betrachtet. Sie greift auf die geschichtlichen Ereignisse zurück, die Realität der Protagonistin prägten. Die autobiographische Spurensuche umfasst drei Zeitebenen: die Zeit des Partisanenkampfs während des Zweiten Weltkrieges, die Kindheit und Jugend der Ich-Erzählerin in den 60er und 70er Jahren und die in der Gegenwart zu verortende Ebene des Erzählens. Als Intellektuelle setzte sich die Autorin mit der Tatsache auseinander, dass in Österreich niemand etwas über die Geschehnisse während des Krieges wusste. Sie beschäftigte sich mit Kontexten, die in der Öffentlichkeit keine Geltung hatten. Für sie

$7 \quad$ Mit einem Ausschnitt aus diesem Roman (Seite 75 - 91) hat die Autorin den Ingeborg-Bachmann-Preis 2011 gewonnen.

8 Die Autorin wuchs mit den Geschichten ihrer Familie und Nachbarn auf, die sie die ganze Zeit beunruhigt haben. Sie ist mit dem Gefühl aufgewachsen, ihre Existenz in der gesamten Zeit bestätigen zu müssen. 
waren das Dinge, die sie zum Nachdenken und zur Reflexion ermutigten. Ihr Zweck war es, poetisch über die Geschichte zu schreiben und ihre breiteren sowie tieferen Dimensionen zu untersuchen.

Im Werk konnten fünf geschichtsphilosophische Konzepte entschlüsselt werden. Bei der zyklischen Wiederholung sind das Geheimnis der Bedrohtheit des Menschen und der Einfluss der Erinnerungen aus der Kindheit bedeutsam. Die Protagonistin fühlt eine innerliche Unruhe in sich und glaubt darüber mit niemandem reden zu können. Dieser Teil von ihr macht ihre Intimität aus, die schmerzhaft ist. Auch das Konzept der Einzigartigkeit und der Unwiederholbarkeit der Geschichte ist für das Werk von großer Bedeutung. Martina Wunderer, Doktorandin der Vergleichenden Literaturwissenschaft, ist der Meinung, dass die politischen Verhältnisse in Kärnten auf das private, persönliche Leben eine Auswirkung haben. Konkret wird das im Roman durch das Leiden der slowenischen Minderheit und die Familiengeschichte der Erzählerin thematisiert. Hierbei sind die Reformen im Schulsystem in Kärnten, die Problematik der Ortstafelfrage und der Grenze zu erwähnen:

Ist der Friede sichtbar geworden? Kann ein slowenischer Ortsname neben einem deutschen Ortsnamen stehen, mehrdeutiger als eine Friedenstaube, ein Regenbogen, ein Monument? (EdV, 220)

Das Problem der Grenze und der Zugehörigkeit in Verbindung mit der Sprache wird ebenso problematisiert:

Der Grenze wegen, die in den Augen der Mehrheit in unserem Lande nur eine nationale und so sprachliche Grenze sein kann, muss ich mich erklären und ausweisen. Wer ich bin, zu wem ich gehöre, warum ich Slowenisch schreibe oder Deutsch spreche? (EdV, 220) ${ }^{9}$

Die Geschichte kann auch als Parabel betrachtet werden, weil es eine gleichnishafte belehrende Erzählung für den Leser ist. Der Leser lernt viel über das alltägliche Leben der Kärntner Slowenen, z. B. dass es der Bevölkerung nicht möglich war, das slowenische Fernsehen offiziell zu empfangen. Auch das Sich-Vertrautmachen mit dem Fremden ist der Schüssel zu der wahren Erkenntnis der Geschichte. Dazu gehören die Kriegsgeschichten der Großmutter. Eine davon war die Rückkehr der Frauen nach Hause. Der Roman bietet auch einen Blick auf die Bildung und Lehre der Großmutter, sowie auf die moralische, religiöse und irrationale Geschichtsdeutung. Ein Beispiel für die Irrationalität wäre, wenn die Großmutter über die Nacht

9 Bei der Untersuchung der Geschichte ihrer Familie faszinierte sie das Bedürfnis nach Erhaltung der slowenischen Identität, vor allem der slowenischen Sprache (Vrščaj, Tina: Angel pozabe Maje Haderlap. Smrt, ki se kratkočasi na Koroškem. In: Pogledi.si. 2012) 
eine Schale Milch auf den Tisch für die Toten stellt. Die Erinnerungen an die Zeit des Krieges stehen in Verbindung mit Angst, Hunger und Tod. Die Geschichte kann auch als Parodie gedeutet werden. Als komisch-satirisch wirk die Aufbewahrung ... und die Erinnerung an den Großvater.

\section{SCHLUSSFOLGERUNG}

„Die Literatur ist schon immer die wirksamste Waffe für die Überwindung der politischen und ideologischen Grenzen gewesen. Sie hat sie immer besiegt. Aber jetzt kommt es zu einem neuen Phänomen. Bemerkbarer werden Autoren, deren Muttersprache nicht Deutsch ist, oder die Sprache der Mehrheit. Vorher hat die nationale Literatur dominiert. Jetzt öffnen sich diese Räume. Deswegen müssen sie anders interpretiert und offener bewertet und betrachtet werden," meint Maja Haderlap (Soban, Branko, 2014: Angel pozabe na Manhattnu. In: Delo, Nr. 52). Sie hat mit ihren poetischen Erinnerungen an die Kindheit und das Erwachsenwerden die verschwundene und archaische Welt, die nicht nur von der Modernität, sondern mehr von der Assimilation bedroht ist, beschrieben.

Die Geschichte ist eine authentische Erzählung einer erwachsenen Frau, die sich in ihre Kindheit begibt und sie, ausgestattet mit dem Wissen von heute, als Gegenwart erlebt. Die Ich-Erzählerin können wir mit der Autorin verbinden. Die Problematik ist die Diskriminierung der Kärntner Slowenen. Die Minderheit wurde schlecht behandelt und hatte keine Rechte. Eindringlich, poetisch, mit einer bezaubernden Unmittelbarkeit fand die Autorin eine Sprache dafür. Ausgerechnet der unbedingte Ausdruckswille und das Bemühen um poetische Dichte führen gegen Ende zu einer überbordenden, prätentiösen Bildhaftigkeit, die Walter Benjamins Thesen zum Begriff der Geschichte entlehnt ist (Struck, Lothar, 2011: Ein immergültiges Denkmal. In: Glanz \& Elend. Magazin für Literatur und Zeitkritik):

Der Engel der Geschichte wird über mich geflogen sein. Seine Flügel werden einen Schatten auf das Lagergelände geworfen haben. Ich habe sein entsetztes Antlitz im Halbdunkel nicht sehen können, nur kurz geglaubt, einen Flügelschlag gehört zu haben, einen Windstoß mit seinen Engelsflügeln vernommen zu haben, in denen sich die Stürme des Kommenden verfingen. (EdV, 286)

In diesem Beispiel zeigt sich die Intertextualität, die die Vermittlung der grausamen Geschehnisse zu übertönen versucht. Es geht um die Relevanz der historischen und politischen Realität, die man als Fiktion ausschildert. Die individuelle Lebensgeschichte 
wird durch Gefühle, Erinnerungen und Traumsequenzen geprägt. Es ist ein wichtiges Buch, weil es zum einen die historische und reale Verankerung von fiktionalen Gefühlswelten anzeigt, die aus dem individuellen sowie kollektiven Gedächtnis entstehen und Selbst- und Fremdbilder prägen, und zum anderen die Mühen und Herausforderungen der Versprachlichung von Gewaltgeschichte veranschaulicht. Maja Haderlap gelingt es mit ihrem Engel des Vergessens das kollektive Gedächtnis der Kärntner Slowenen in den Vordergrund zu stellen.

Das Buch ist ein literarisches Denkmal für die slowenische Minderheit. Maja Haderlap hat eine literarische wie politische Stimme, die durch Engel des Vergessens zu den wichtigsten der Literatur-Landschaft Österreichs gehört.

\section{LITERATURVERZEICHNIS}

BHABHA, Homi. K (1994) The Location of Culture. New York: Routledge. BOGATAJ, Matej. (2013) Maja Haderlap, Angel pozabe. Mladina 35,72.

BORLONI, Bergit (2012) Eine Geschichte gegen das Vergessen und über das Nachwirken von Traumata. (Belletristik-couch.de.: http:/www.belletristik-couch.de/maja-haderlap-engel-des-vergessens.html, letzter Zugriff: 12. September 2014).

BOROVNIK, Silvija (2012) Dvojezičnost slovenske književnosti v Avstriji kot odraz dvojne identitete. Individualna in kolektivna dvojezičnost. Ljubljana: Znanstvena založba Filozofske fakultete, 437 - 448.

BOROVNIK, Silvija (2012) Dvojna identiteta slovenske književnosti v Avstriji in roman Maje Haderlap Angel pozabe (2011). Večno mladi Htinj: ob 80-letnici Janka Čara. Hg. Marko Jesenšek. Maribor: Mednarodna založba Oddelka za slovanske jezike in književnosti, Filozofska fakulteta, 187 - 199.

GEPPERT, Vilmar Hans (1976) Der »andere« historische Roman. Theorie und Strukturen einer diskontinuierlichen Gattung. Tübingen: Niemeyer.

GEPPERT, Vilmar Hans (2009) Der Historische Roman: Geschichte umerzählt von Walter Scott bis zur Gegenwart. Tübingen: A. Francke Verlag.

GLAZ, Agata (2014) Tema nasilja totalitarnih režimov v literarnih besedilih nekaterih slovenskih pisateljic in publicistk. Jezik in Slovstvo, 59, $43-52$.

GREINER, Ulrich (2011) Gerechtigkeit für die Slowenen. (Zeit Online. 20. September 2014. http://www.zeit.de/2011/30/L-Haderlap\#,).

GROSMAN, Meta (2004) Književnost v medkulturnem položaju. Ljubljana: Znanstveni inštitut Filozofske fakultete.

HADERLAP, Maja (2011) Engel des Vergessens. Göttingen: Wallstein Verlag. HELL, Cornelius (2011) Der Engel des Vergessens. Romandebüt von Maja Haderlap. (OE1.ORF. 12. September 2014. http://oe1.orf.at/artikel/283580.). 
HÖBEL, Wolfgang. (2011) Im Keller summen die Bienen. Der Spiegel 29, 125.

HOFMANN, Michael (2006) Interkulturelle Literaturwissenschaft. Eine Einführung. Padeborn: W. Fink UTB Verlag.

HORVAT, Marjan (2011) Maja Haderlap: »Rasla sem z občutkom, da moram ves čas dokazovati svojo eksistenco«. (Mladina. 12. September 2014. http://www.mladina.si/86695/maja-haderlap-rasla-sem-z-obcutkom-da-moram-ves-cas-dokazovati-svojo-eksistenco/.).

HUMPHREY, Richard (1986) The Historical Novel as Philosophy of History. Three German Contributions: Alexis, Fontane, Döblin.. (Bithell Series of Dissertations, 10). London: Institute of Germanic Studies (University of London).

JEZERNIK, Jerneja(2011)Angel pozabejepostalmojapripoved.(Pogledi.si.21. September 2014. http://www.pogledi.si/ljudje/angel-pozabe-je-postal-moja-pripoved,).

KONDRIČ HORVAT, Vesna (2000) Fiktionalisierung der eigenen Biographie durch Sprachexperimente zu Erica Pedretti. Acta neophilologica, 33. 35 - 47.

KONDRIČ HORVAT, Vesna (2012) Transkulturelle Aspekte in Erica Pedrettis Roman Engste Heimat. Hg. Meike Penkwitt, Die Erinnerungstexte der Autorin Erica Pedretti. Würzburg: Königshausen \& Neumann, 131-142.

KOS, Dejan (2003) Theoretische Grundlage der empirischen Literaturwissenschaft. Maribor: Slavistično društvo Zora.

MAYER, Norbert (2012) Haderlap: Deutsch hält mich auf Distanz zum Schmerz. (Die Presse.com. 12. September 2014. http://diepresse.com/home/kultur/ literatur/743059/Haderlap_Deutsch-haelt-mich-auf-Distanz-zum-Schmerz).

MÜLLER, Harro (1988) Zwischen Kairos und Katastrophe. Historische Romane im 20. Jahrhundert. Frankfurt am Main: Athenäums Monographien Literaturwissenschaft. NÜNNING, Angsar (1995) Von historischer Fiktion zu historiographischer Metafiktion. Teil I: Theorie, Typologie und Poetik des historischen Romans. Trier: Wissenschaftlicher Verlag.

NÜNNING, Ansgar (2001) Metzler Lexikon Literatur- und Kulturtheorie. Ansätze, Personen, Grundbegriffe. 2. überarbeitete und erweiterte Aufl. Stuttgart/Weimar: J. B. Metzler.

ORF KÄRNTEN (2011) 35. Tage der deutschsprachigen Literatur. (Bachmannpreis. 12. September 2014. http://bachmannpreis.eu/de/information/3696).

RACHINGER, Johanna (2012) Engel des Vergessens - Von Maja Haderlap. (Kurier. 12. September 2014. http://kurier.at/kultur/engel-des-vergessens-von-maja-haderlap/810.686)

ŠLIBAR, Neva (2009) RundUm Literatur I. Der literarische Text. Ljubljana: Znanstvena založba.

ŠLIBAR, Neva (2012) Gefühlswelten und Emotionsdiskurse in Literatur und Literaturdidaktik. Gefühlswelten in der fremdsprachlichen Didaktik. Ljubljana: Znanstvena založba Filozofske fakultete. 
SOBAN, Branko. (2014) Angel pozabe na Manhattnu. Delo 52.

STRUCK, LOTHAR (2011) Ein immergültiges Denkmal. (Glanz \& Elend. Magazin für Literatur und Zeitkritik. 12. September 2014. http://www.glanzundelend.de/ Artikel/abc/h/maja_haderlap.htm).

STRUTZ, Johann/Peter ZIMA V. (1996) Literarische Polyphonie. Übersetzung und Mehrsprachigkeit in der Literatur. Tübingen: Günter Narr Verlag.

VRŠČCA, Tina (2012) Angel pozabe Maje Haderlap. Smrt, ki se kratkočasi na Koroškem. (Pogledi.si. 21. September 2014, http://www.pogledi.si/knjiga/ smrt-ki-se-kratkocasi-na-koroskem)

WALLSTEIN, Verlag (2011) Maja Haderlap. Engel des Vergessens. (http://www. wallstein-verlag.de/9783835309531-maja-haderlap-engel-des-vergessens.html. 12. September 2014).

WIERLACHER, Alois (1985) Mit fremden Augen oder: Fremdheit als Ferment. Überlegungen zur Begründung einer interkulturellen Hermeneutik deutscher Literatur. Das Fremde und das Eigene. Prolegomena zu einer interkulturellen Germanistik. München, $51-80$.

WINKEL, Reiner (2005) Am Anfang war die Hure. Theorie und Praxis der Bildung oder: Eine Reise durch die Geschichte des Menschen - in seinen pädagogischen Entwürfen. Baltmannsweiler: Schneider- Verlag Hohengehren.

\section{ABSTRACT}

\section{Authenticity in Engel des Vergessens (Angel of Oblivion), a novel by Maja Haderlap}

This article analyses the authenticity of memories in the second half of the 20th century in Carinthia. The segmentation of historical authenticity is visible in the historical reminiscence of traumatic historical events in relation to social interaction. The combination of social, political und cultural elements affects the divergent experience of individuals in determinated environment. The complexity of the novel is seen in the inner feelings, which characterization relates to emotions and the subconscious of the population. The article focuses on the female perspective as well as on the role of women in the story. The author proves with her experience of unconsciously the unsolved problematic of her own literalizing of history. The personality of the writer is shown in the examples of double literary, personal and lingual identity.

Keywords: literalizing of history, the role of women/ multiculturalism/ the search for identity/ the question of minority 


\section{POVZETEK}

\section{Avtentičnost v romanu Maje Haderlap Angel pozabe}

Članek poskuša na podlagi romana Angel pozabe pisateljice Maje Haderlap prikazati avtentičnost spominov doživljanja dogodkov na avstrijskem Koroškem v drugi polovici dvajsetega stoletja. Segmentacija zgodovinske avtentičnosti je vidna v obujanju travmatičnih doživetij, ki imajo sledove v preteklosti in medosebnih odnosih. Preplet socialnih, političnih ter kulturnih elementov vpliva na različne izkušnje posameznikov v determiniranem okolju. Kompleksnost romana je vidna v notranjih občutkih figur, katerih karakterizacija se navezuje na emocije in podzavest prebivalstva. Roman je obravnavan iz perspektive ženske zgodbe in proučuje vlogo ženske v romanu. Avtorica preko svojega umetniškega besedila s pridihom poetičnosti in svojim podzavestnim doživljanjem dokazuje nerazrešenost problematike lastne literarizacije zgodovine. Osebnost pisateljice se izkazuje v primerih dvojnih literarnih, osebnih in jezikovnih identitet.

Ključne besede: literarizacija zgodovine, vloga ženske, multikulturnost, iskanje identitete, vprašanje manjšine 